\title{
THE MOTION OF A LARGE PARTICLE $\left({ }^{1}\right)$
}

\author{
BY \\ RICHARD HOLLEY
}

1. Introduction. This paper resulted from an attempt to find a probabilistic model for the motion of a large heavy particle colliding with many light particles. The physical situation is as follows. Consider a one-dimensional world in which there are countably many particles distributed at random and each moving independently of the others except for collisions. T. E. Harris [3] has given very general conditions under which the paths of the particles in such a system are well defined. In our model the particles will move with constant velocity until they collide with other particles. In this case if the particles do not have any accumulation points initially, and if their initial velocities are uniformly bounded, then the paths will be well defined. We will assume that all collisions are perfectly elastic, conserving momentum and energy; therefore, when two particles with the same mass collide, they simply exchange velocities. Now the problem that we will be concerned with is to describe the behavior of a particle which is much heavier than the others.

Before beginning on this problem, consider a system as described above in which particles of equal mass are started at all of the even integers, and each is independently given a velocity of \pm 1 with probability $1 / 2$. We will consider the one which started at zero as a distinguished particle. It is clear that if we observe this particle after every unit time, it will be performing a simple random walk. However, more than this is true. If we denote by $X(t)$ the position of our distinguished particle at time $t$, the following theorem is true. (See Billingsley [1, page 68] for the proof.)

1.1 Theorem. Consider the sequence of measures $\left\{\mu_{A}\right\}$, on $C[0,1]$ (=continuous functions on $[0,1])$ induced by $X_{A}(t)=X(A t) / \sqrt{ } A$. As $A$ goes to infinity, $\mu_{A}$ converges weakly to the Wiener measure.

The reader unfamiliar with this notion of convergence is referred to Billingsley [1, page 11].

Various authors (D. W. Jepsen [5], T. E. Harris [3], and others) have studied the above model and generalizations of it. F. Spitzer [8] has proved that if, instead of

Received by the editors February 26, 1969.

(1) This paper was prepared with the support of the National Science Foundation through a Graduate Fellowship at Cornell University. The author wishes to thank Professor F. Spitzer for suggesting this problem and for the many valuable contributions that he made towards its solution. 
starting the particles in the even integers, they are started in a Poisson $\left({ }^{2}\right)$ process with density one and given velocities which are independent and equidistributed with mean zero, then the same results hold.

In the problem where the distinguished particle is heavier it is clear that we have one more parameter to vary than in the above examples: the mass of the distinguished particle. We will let the light particles start out in a Poisson process with density one, and give them independently velocities \pm 1 with probability $1 / 2$. We then put a particle at the origin with mass $M$ times as great as the others. Let $Z_{M}(t)$ denote the position of the heavy particle at time $t$ and $Y_{M}(t)=Z_{M}(M t) / \sqrt{ } M$. In this notation (physically perhaps not the most natural one as pointed out below) we can state one of the main results of this paper.

1.2 Theorem. As $M$ goes to infinity the family of measures, $\left\{v_{M}\right\}$, induced on $C[0,1]$ by $\left\{Y_{M}\right\}$, converges weakly to the measure corresponding to the OrnsteinUhlenbeck position process (as defined by Nelson [6, §9]).

Instead of changing the time and space scales in the above problem, we may produce the same effect by increasing the density of the Poisson process (i.e. the expected number of particles per unit length) and increasing the velocity of the lighter particles. This interpretation is more physically meaningful and is the one we will use. It turns out that the square of the velocity of the lighter particles must increase at the same rate as the ratio of the masses in order to get a nondegenerate limit for the velocity process.

The motivation behind the choice of the Poisson process is as follows. Stone [9] and Dobrušin [2] have proved theorems to the effect that if each particle in a system of particles, which is not too badly behaved originally, is allowed to undergo a motion independent of the others, then as the time gets large the system looks more and more like a Poisson process. The motion of the lighter particles described above does not satisfy the hypotheses of these theorems, but if it were very much more random it would. Other motivating factors are the well known fact that if the heavy particle were not present the system would remain in a Poisson process, and the fact that the Poisson process is in some sense the most random. (See Renyi [7].)

Theorem 1.2 is still true if the initial velocities of the lighter particles are taken from a much more general distribution than we will deal with here (see Holley [4]). However, it has so far not been possible to generalize the results to two or more dimensions.

2. The main results. First we set some notations which we will use in this section.

Let $(\Omega, \mathscr{F}, P)$ be a probability space on which there is defined an OrnsteinUhlenbeck velocity process, $X(t)$, that starts at zero. That is, $P(X(0)=0)=1$, and

$\left.{ }^{2}\right)$ The number of particles in any bounded interval is a Poisson random variable with mean equal to the density times the length of the interval, and the numbers in disjoint intervals are independent. 
the transition function is given by

$$
Q_{t}\left(v_{0}, d v\right)=\frac{1}{(2 \pi)^{1 / 2} \sigma(t)} \exp \left(-\left[\frac{\left(v-\alpha(t) \dot{v}_{0}\right)^{2}}{2 \sigma^{2}(t)}\right]\right) d v
$$

(where $\sigma^{2}(t)=D\left(1-e^{-2 \beta t}\right) / 2 \beta$ and $\left.\alpha(t)=e^{-\beta t}\right)$.

Let $D[0,1]$ be the space of right continuous functions with left-hand limits on $[0,1]$, and give $D[0,1]$ the Skorohod topology.

Let $Z: \Omega \rightarrow D[0,1]$ be defined by $Z(\omega)(t)=X(t, \omega)$. Then $Z$ induces a measure $P^{\prime}$ on $D[0,1]$ by the formula $P^{\prime}(A)=P\left(Z^{-1}(A)\right)$. In the future we will drop the prime from the notation when this will cause no difficulty.

Now consider a Poisson process of "atoms" on the real line with density $\lambda(M)$ and mass one, and a big "molecule" sitting still at the origin. Each of the atoms is independently given a velocity $\pm v(M)$ each with probability $1 / 2$, and the system is allowed to undergo collisions conserving momentum and energy. That is, if the molecule has mass $M$ and velocity $V_{1}$ and is hit by an atom with velocity $v_{1}$, then the equations

$$
M V_{1}+v_{1}=M V_{2}+v_{2} \text { and } M V_{1}^{2}+v_{1}^{2}=M V_{2}^{2}+v_{2}^{2}
$$

are both satisfied. Here $V_{2}$ and $v_{2}$ are the velocities after the collision. Solving these equations we get

$$
V_{2}=\frac{M-1}{M+1} V_{1}+\frac{2}{M+1} v_{1} \quad \text { and } \quad v_{2}=-\frac{M-1}{M+1} v_{1}+\frac{2 M}{M+1} V_{1} .
$$

Let $V_{M}(t)$ be the velocity of the molecule at time $t$ if the atoms are removed after a collision with the molecule, and let $V_{M}^{\prime}(t)$ be the velocity at time $t$ when the atoms are not removed. We will denote the measure which $V_{M}$ induces on $D[0,1]$ by $P_{M}$ and the measure which $V_{M}^{\prime}$ induces on $D[0,1]$ by $P_{M}^{\prime}$.

From (2.1) it is seen that when two atoms collide, they simply exchange velocities; and hence, we may assume that they just pass through each other. Also since the molecule never gets going faster than $v(M)$, it cannot hit any atom which is initially moving away from it. Hence we will assume that the density of the Poisson process is $\lambda(M) / 2$, and that at time zero all atoms are moving toward the molecule with speed $v(M)$.

To simplify calculations we will first assume that the atoms are removed from the system after a collision with the molecule. This assumption will be removed later. Now using (2.1) and the fact that the distances between atoms are independent exponential random variables with mean $2 / \lambda(M)$, it is a routine matter to check that $V_{M}(t)$ is a Markov process which can be described as follows. If the molecule has velocity $V$, it maintains that velocity for an exponentially distributed time with mean $1 / \lambda(M) v(M)$ and then jumps to

$$
\begin{aligned}
& \frac{M-1}{M+1} V+\frac{2}{M+1} v(M) \quad \text { with probability } \frac{v(M)-V}{2 v(M)} \text { or } \\
& \frac{M-1}{M+1} V-\frac{2}{M+1} v(M) \quad \text { with probability } \frac{v(M)+V}{2 v(M)} .
\end{aligned}
$$


Now let $X_{M}(k)$ be a Markov chain whose one step transition function is given by (2.2), and let $N_{M}(t)$ be a Poisson random variable with mean $t \lambda(M) v(M)$, which is independent of $X_{M}(k)$. Then it is clear that for any real-valued function $f$,

$$
E\left\{f\left(V_{M}(t+s)\right) \| V_{M}(s)\right\}=E\left\{f\left(X_{M}\left(N_{M}(t)\right)\right) \| X_{M}(0)=V_{M}(s)\right\} .
$$

Here $E\{\|\}$ denotes conditional expectation.

We are now ready to use Theorem 19.4 of Billingsley [1, p. 164] and his remarks on page 165 to prove the following

2.1 TheOREM. Let $v(M)=((M+1) D / \beta)^{1 / 2}$ and $\lambda(M)=(\beta / 4)((M+1) \beta / D)^{1 / 2}$. Then as $M \rightarrow \infty, P_{M}$ converges weakly to $P$.

Proof. According to Billingsley's Theorem 19.4, if we can show that

(i) $P_{M}\left(V_{M}(0)=0\right)=1$;

(ii) $P_{M}\left(\sup _{0 \leqq t \leqq 1}\left|V_{M}(t)-V_{M}(t-)\right| \leqq \varepsilon_{M}\right)=1$ with $\varepsilon_{M} \rightarrow 0$;

(iii) $\lim _{h \rightarrow 0} \lim \sup _{M \rightarrow \infty}(1 / h) E\left\{\left|E\left\{V_{M}(t+h)-V_{M}(t) \| V_{M}(t)\right\}+h \beta V_{M}(t)\right|\right\}=0$, $\lim _{h \rightarrow 0} \lim \sup _{M \rightarrow \infty}(1 / h) E\left\{\left|E\left\{\left(V_{M}(t+h)-V_{M}(t)\right)^{2} \| V_{M}(t)\right\}-D h\right|\right\}=0$;

(iv) there exists a constant $K$ such that if $t_{1} \leqq t \leqq t_{2}$,

$$
E\left\{\left(V_{M}\left(t_{1}\right)-V_{M}(t)\right)^{2}\left(V_{M}(t)-V_{M}\left(t_{2}\right)\right)^{2}\right\} \leqq K\left(t_{2}-t_{1}\right)^{2}
$$

uniformly in $M$, then $\left\{P_{M}\right\}$ converges weakly to a measure $\mu$. Furthermore, $\mu$ is the measure concentrated on $C[0,1]$ whose finite-dimensional distributions are Gaussian and having

$$
\begin{aligned}
E_{\mu}\{X(t)\} & =0, \quad 0 \leqq t \leqq 1, \\
E_{\mu}\{X(s) X(t)\} & =\int_{0}^{s} D \exp \left(\left[2 \int_{r}^{s}-\beta d t+\int_{s}^{t}-\beta d t\right]\right) d r \\
& =\exp [-\beta(s+t)] D(\exp (2 \beta s)-1) / 2 \beta, \quad 0 \leqq s \leqq t \leqq 1,
\end{aligned}
$$

(i.e. $\mu=P$; see Nelson $[6, \S 9$, p. 55$]$ ).

(i) This is obvious since we always start the molecule with zero velocity.

(ii) First notice that with probability one $\left|V_{M}(t)\right| \leqq v(M)$ for all $t$. Then it is easily seen from (2.1) that if there is a discontinuity at $t$, we will have

$$
\left|V_{M}(t-)-V_{M}(t)\right| \leqq \frac{2}{M+1}\left(\left|V_{M}(t-)\right|+v(M)\right) \leqq \frac{4 v(M)}{M+1} .
$$

Therefore,

$$
P_{M}\left(\sup _{0 \leqq t \leqq 1}\left|V_{M}(t)-V_{M}(t-)\right| \leqq 4 v(M) /(M+1)\right)=1,
$$

and (ii) is proved for

$$
\varepsilon_{M}=4 v(M) /(M+1)=4((M+1) D / \beta)^{1 / 2} /(M+1) \rightarrow 0 .
$$

In order to prove (iii) and (iv) we need two lemmas. 
2.2 Lemma. Let $X_{M}(k)$ be the Markov chain whose one step transition is given by (2.2), and $M \geqq 10$. Then

(a) $E\left\{X_{M}(k) \| X_{M}(0)\right\}=A_{1}^{k}(M) X_{M}(0)$,

(b) $E\left\{X_{M}^{2}(k) \| X_{M}(0)\right\}=B_{2}(M)(D / \beta)\left(1-B_{1}^{k}(M)\right)+B_{1}^{k}(M) X_{M}^{2}(0)$,

(c) $E\left\{X_{M}^{3}(k) \| X_{M}(0)\right\}=C_{1}^{k}(M) X_{M}^{3}(0)+(D / \beta) C_{2}(M)\left(A_{1}^{k}(M)-C_{1}^{k}(M)\right) X_{M}(0)$, $E\left\{X_{M}^{4}(k) \| X_{M}(0)\right\}=F_{1}^{k}(M) X_{M}^{4}(0)+(D / \beta) F_{2}(M)\left\{B_{1}^{k}(M)-F_{1}^{k}(M)\right\} X_{M}^{2}(0)$

(d)

$$
\begin{aligned}
& +(D / \beta)^{2} F_{3}(M) B_{2}(M)\left\{1-F_{1}^{k}(M)\right\} \\
& -(D / \beta)^{2} F_{2}(M) B_{2}(M)\left\{B_{1}^{k}(M)-F_{1}^{k}(M)\right\} \\
& +(D / \beta)^{2} F_{4}(M)\left\{1-F_{1}^{k}(M)\right\},
\end{aligned}
$$

where

$$
\begin{aligned}
& A_{1}(M)=\frac{M-3}{M+1} \\
& B_{1}(M)=\frac{M-1}{M+1} \frac{M-5}{M+1} \\
& B_{2}(M)=\frac{M+1}{2 M-1} \\
& C_{1}(M)=\left(\frac{M-1}{M+1}\right)^{2} \frac{M-7}{M+1} \\
& C_{2}(M)=\frac{(M+1)(3 M-5)}{2 M^{2}-5 M+1} \\
& F_{1}(M)=\left(\frac{M-1}{M+1}\right)^{3} \frac{M-9}{M+1} \\
& F_{2}(M)=\frac{6 M^{2}-8 M-14}{2 M^{2}-7 M+1} \\
& F_{3}(M)=\frac{3 M^{3}-7 M^{2}-3 M+7}{2 M^{3}-3 M^{2}+4 M-1} \\
& F_{4}(M)=\frac{2(M+1)^{2}}{2 M^{3}-3 M^{2}+4 M-1}
\end{aligned}
$$

The restriction $M \geqq 10$ is just for convenience so that we do not have to worry about any of these coefficients being infinite.

Proof. Let $M$ be fixed; we will suppress it from the notation during the proof.

(a) One easily checks that

$$
E\{X(k) \| X(k-1)\}=\frac{M-3}{M+1} X(k-1)=A_{1} X(k-1),
$$

and the result follows by induction. 
(b) First one checks that

$$
\begin{aligned}
E\left\{X^{2}(k) \| X(k-1)\right\} & =\frac{M-1}{M+1} \frac{M-5}{M+1} X^{2}(k-1)+\frac{4}{M+1} \frac{D}{\beta} \\
& =B_{1} X^{2}(k-1)+\frac{4}{M+1} \frac{D}{\beta} .
\end{aligned}
$$

We now proceed by induction. For $k=0$ the result is obvious. Assume that (b) is true for $k-1$. Then

$$
\begin{aligned}
E\left\{X^{2}(k) \| X(0)\right\} & =E\left\{E\left\{X^{2}(k) \| X(k-1)\right\} \| X(0)\right\} \\
& =E\left\{B_{1} X^{2}(k-1)+\frac{4}{M+1} \frac{D}{\beta} \| X(0)\right\} \\
& =B_{1}\left\{B_{2} \frac{D}{\beta}\left(1-B_{1}^{k-1}\right)+B_{1}^{k-1} X^{2}(0)\right\}+\frac{4}{M+1} \frac{D}{\beta} \\
& =\frac{D}{\beta}\left(B_{1} B_{2}-B_{1}^{k} B_{2}+\frac{4}{M+1}\right)+B_{1}^{k} X^{2}(0) .
\end{aligned}
$$

Now one simply checks that

$$
B_{1} B_{2}+4 /(M+1)-B_{1}^{k} B_{2}=B_{2}\left(1-B_{1}^{k}\right) .
$$

(c) First we check that

$$
E\left\{X^{3}(k) \| X(k-1)\right\}=C_{1} X^{3}(k-1)+\frac{D}{\beta} \frac{12 M-20}{(M+1)^{2}} X(k-1) .
$$

Again we proceed by induction on $k$. For $k=0$ the result is obvious. Assume that (c) is true for $k-1$. Then

$$
\begin{aligned}
E\left\{X^{3}(k) \| X(0)\right\}= & E\left\{E\left\{X^{3}(k) \| X(k-1)\right\} \| X(0)\right\} \\
= & C_{1} E\left\{X^{3}(k-1) \| X(0)\right\}+\frac{D}{\beta} \frac{12 M-20}{(M+1)^{2}} E\{X(k-1) \| X(0)\} \\
= & C_{1}\left\{C_{1}^{k-1} X^{3}(0)+\frac{D}{\beta} C_{2}\left(A_{1}^{k-1}-C_{1}^{k-1}\right) X(0)\right\} \\
& +\frac{D}{\beta} \frac{12 M-20}{(M+1)^{2}} A_{1}^{k-1} X(0) \\
= & C_{1}^{k} X^{3}(0)+\frac{D}{\beta}\left\{C_{1} C_{2} A_{1}^{k-1}+\frac{12 M-20}{(M+1)^{2}} A_{1}^{k-1}-C_{2} C_{1}^{k}\right\} X(0) .
\end{aligned}
$$

Now part (c) is completed by checking that

$$
C_{1} C_{2} A_{1}^{k-1}+\frac{12 M-20}{(M+1)^{2}} A_{1}^{k-1}=C_{2} A_{1}^{k}
$$

(d) Again we check that

$$
E\left\{X^{4}(k) \| X(k-1)\right\}=F_{1} X^{4}(k-1)+(D / \beta) G X^{2}(k-1)+(D / \beta)^{2} H
$$


where

$$
G=\frac{8}{(M+1)^{3}}\left[3(M-1)^{2}-4(M-1)\right], \quad H=\left(\frac{4}{M+1}\right)^{2} .
$$

Notice that it will be sufficient to prove that

$$
\begin{aligned}
E\left\{X^{4}(k) \| X(0)\right\}= & F_{1}^{k} X^{4}(0)+\frac{D}{\beta} G \frac{B_{1}^{k}-F_{1}^{k}}{B_{1}-F_{1}} X^{2}(0) \\
& +\left(\frac{D}{\beta}\right)^{2} G B_{2}\left(\frac{1-F_{1}^{k}}{1-F_{1}}-\frac{B_{1}^{k}-F_{1}^{k}}{B_{1}-F_{1}}\right)+\left(\frac{D}{\beta}\right)^{2} H \frac{1-F_{1}^{k}}{1-F_{1}},
\end{aligned}
$$

since one can easily check that

$$
G /\left(B_{1}-F_{1}\right)=F_{2}, \quad G /\left(1-F_{1}\right)=F_{3}, \quad \text { and } H /\left(1-F_{1}\right)=F_{4} .
$$

Once again we proceed by induction on $k$. For $k=0$ the result is obvious. Assume that (d) is true for $k-1$. Then

$$
\begin{aligned}
E\left\{X^{4}(k) \| X(0)\right\}=E\{ & \left.E\left\{X^{4}(k) \| X(k-1)\right\} \| X(0)\right\} \\
= & E\left\{F_{1} X^{4}(k-1)+(D / \beta) G X^{2}(k-1)+(D / \beta)^{2} H \| X(0)\right\} \\
= & F_{1}\left[F_{1}^{k-1} X^{4}(0)+\frac{D}{\beta} G \frac{B_{1}^{k-1}-F_{1}^{k-1}}{B_{1}-F_{1}} X^{2}(0)\right. \\
& \left.\quad+\left(\frac{D}{\beta}\right)^{2} G B_{2}\left(\frac{1-F_{1}^{k-1}}{1-F_{1}}-\frac{B_{1}^{k-1}-F_{1}^{k-1}}{B_{1}-F_{1}}\right)+\left(\frac{D}{\beta}\right)^{2} H \frac{1-F_{1}^{k-1}}{1-F_{1}}\right] \\
& \quad+\frac{D}{\beta} G\left\{B_{2} \frac{D}{\beta}\left(1-B_{1}^{k-1}\right)+B_{1}^{k-1} X^{2}(0)\right\}+\left(\frac{D}{\beta}\right)^{2} H .
\end{aligned}
$$

Upon multiplying through by $F_{1}$ and $(D / \beta) G$ and then regrouping, this last expression is seen to be equal to

$$
F_{1}^{k} X^{4}(0)+\frac{D}{\beta} G\left\{\frac{B_{1}^{k}-F_{1}^{k}}{B_{1}-F_{1}}\right\} X^{2}(0)+\left(\frac{D}{\beta}\right)^{2} G B_{2}\left\{\frac{1-F_{1}^{k}}{1-F_{1}}-\frac{B_{1}^{k}-F_{1}^{k}}{B_{1}-F_{1}}\right\}+\left(\frac{D}{\beta}\right)^{2} H\left\{\frac{1-F_{1}^{k}}{1-F_{1}}\right\}
$$

This concludes the proof of the lemma.

2.3 Lemma. For all $M \geqq 10$ we have

(a) $\quad E\left\{V_{M}(t+h) \| V_{M}(t)\right\}=e^{-\beta h} V_{M}(t)$,

(b) $\quad E\left\{V_{M}^{2}(t+h) \| V_{M}(t)\right\}=e^{-\beta h G_{1}(M)} V_{M}^{2}(t)+B_{2}(M) \frac{D}{\beta}\left\{1-e^{-\beta h G_{1}(M)}\right\}$,

(c) $E\left\{V_{M}^{3}(t+h) \| V_{M}(t)\right\}=e^{-\beta h G_{2}(M)} V_{M}^{3}(t)+(D / \beta) C_{2}(M)\left\{e^{-\beta h}-e^{-\beta h G_{2}(M)}\right\} V_{M}(t)$,

$$
E\left\{V_{M}^{4}(t+h) \| V_{M}(t)\right\}=e^{-\beta h G_{3}(M)} V_{M}^{4}(t)
$$

$$
\begin{aligned}
& +(D / \beta) F_{2}(M)\left\{e^{-\beta h G_{1}(M)}-e^{-\beta h G_{3}(M)}\right\} V_{M}^{2}(t) \\
& +(D / \beta)^{2} F_{3}(M) B_{2}(M)\left\{1-e^{-\beta h G_{3}(M)}\right\} \\
& -(D / \beta)^{2} F_{2}(M) B_{2}(M)\left\{e^{-\beta h G_{1}(M)}-e^{-\beta h G_{3}(M)}\right\} \\
& +(D / \beta)^{2} F_{4}(M)\left\{1-e^{-\beta h G_{3}(M)}\right\} .
\end{aligned}
$$

Here $B_{2}(M), C_{2}(M), F_{2}(M), F_{3}(M), F_{4}(M)$ are as before, and

$$
G_{1}(M)=\frac{2 M-1}{M+1}, \quad G_{2}(M)=\frac{3 M^{2}-3 M+2}{(M+1)^{2}}, \quad G_{3}(M)=\frac{4 M^{3}-6 M^{2}-2}{(M+1)^{3}} .
$$


Proof. Let $M$ be fixed and suppress it from the notation. By (2.3) we have

$$
E\left\{V^{k}(t+h) \| V(t)\right\}=E\left\{X^{k}(N(h)) \| X(0)=V(t)\right\}
$$

where $N(h)$ is a Poisson random variable independent of $X$ with mean

$$
h \lambda v=h(M+1)(\beta / 4) .
$$

To prove (a) notice that

$$
\begin{aligned}
E\{V(t+h) \| V(t)\}= & \exp (-h(M+1) \beta / 4) \sum_{k=0}^{\infty} \frac{1}{k !}\{h(M+1) \beta / 4\}^{k} \\
& \times E\{X(k) \| X(0)=V(t)\} \\
= & \exp (-h(M+1) \beta / 4) \sum_{k=0}^{\infty} \frac{1}{k !}\{h(M+1) \beta / 4\}^{k} A_{1}^{k} V(t) \\
= & \exp \left(-h \beta \frac{M+1}{4}\left(1-A_{1}\right)\right) V(t) .
\end{aligned}
$$

But $1-A_{1}=1-(M-3) /(M+1)=4 /(M+1)$. Therefore,

$$
E\{V(t+h) \| V(t)\}=e^{-\beta h} V(t) .
$$

The proofs of (b), (c), and (d) are the same and will be left to the reader. Notice that

$$
G_{1}(M)=\frac{M+1}{4}\left(1-B_{1}(M)\right), \quad G_{2}(M)=\frac{M+1}{4}\left(1-C_{1}(M)\right),
$$

and

$$
G_{3}(M)=\frac{M+1}{4}\left(1-F_{1}(M)\right)
$$

Returning now to (iii), we have

$$
E\left\{\left|E\left\{V_{M}(t+h)-V_{M}(t) \| V_{M}(t)\right\}+\beta h V_{M}(t)\right|\right\}=E\left\{\left|V_{M}(t) \| e^{-\beta h}-1+\beta h\right|\right\} .
$$

But $(1 / h)\left|e^{-\beta h}-1+\beta h\right| \rightarrow 0$ as $h \rightarrow 0$, and therefore to complete the first part of (iii) it is sufficient to show that $E\left\{\left|V_{M}(t)\right|\right\}$ remains bounded as $M \rightarrow \infty$. This follows from

$$
\begin{aligned}
E\left\{\left|V_{M}(t)\right|\right\} & \leqq 1+E\left\{V_{M}^{2}(t)\right\}=1+E\left\{E\left\{V_{M}^{2}(t) \| V_{M}(0)\right\}\right\} \\
& =1+B_{2}(M) \frac{D}{\beta}\left[1-e^{-\beta t G_{1}(M)}\right] \rightarrow 1+\frac{D}{2 \beta}\left(1-e^{-2 \beta t}\right),
\end{aligned}
$$

since $B_{2}(M) \rightarrow 1 / 2$ and $G_{1}(M) \rightarrow 2$. For the second part of (iii) we have

$$
\begin{aligned}
E\{\mid E\{ & \left.\left.\left(V_{M}(t+h)-V_{M}(t)\right)^{2} \| V_{M}(t)\right\}-D h \mid\right\} \\
& =E\left\{\left|e^{-\beta h G_{1}(M)} V_{M}^{2}(t)+B_{2}(M) \frac{D}{\beta}\left(1-e^{-\beta h G_{1}(M)}\right)-2 e^{-\beta h} V_{M}^{2}(t)+V_{M}^{2}(t)-D h\right|\right\} \\
& \leqq\left|B_{2}(M) \frac{D}{\beta}\left(1-e^{-\beta h G_{1}(M)}\right)-D h\right|+E\left\{V_{M}^{2}(t)\right\}\left|e^{-\beta h G_{1}(M)}-2 e^{-\beta h}+1\right| .
\end{aligned}
$$


As $M \rightarrow \infty$ this last expression approaches

$$
\left|(D / 2 \beta)\left(1-e^{-2 \beta h}\right)-D h\right|+(D / 2 \beta)\left(1-e^{-2 \beta t}\right)\left(e^{-\beta h}-1\right)^{2} .
$$

And therefore,

$$
\begin{aligned}
\lim _{h \rightarrow 0} \limsup _{M \rightarrow \infty} & \frac{1}{h} E\left\{\left|E\left\{\left(V_{M}(t+h)-V_{M}(t)\right)^{2} \| V_{M}(t)\right\}-D h\right|\right\} \\
\leqq & \lim _{h \rightarrow 0} \frac{1}{h}\left\{\left|\frac{D}{2 \beta}\left(1-e^{-2 \beta h}\right)-D h\right|+\frac{D}{2 \beta}\left(1-e^{-2 \beta t}\right)\left(e^{-\beta h}-1\right)^{2}\right\}=0 .
\end{aligned}
$$

To prove (iv) we first notice from Lemma 2.3 that if $M \geqq 10$

$$
\begin{aligned}
& E\left\{V_{M}^{2}(t+h) \| V_{M}(t)\right\}=e^{-\beta h G_{1}(M)} V_{M}^{2}(t)+O_{1}(h), \\
& E\left\{V_{M}^{3}(t+h) \| V_{M}(t)\right\}=e^{-\beta h G_{2}(M)} V_{M}^{3}(t)+O_{2}(h) V_{M}(t),
\end{aligned}
$$

and

$$
E\left\{V_{M}^{4}(t+h) \| V_{M}(t)\right\}=e^{-\beta h G_{3}(M)} V_{M}^{4}(t)+O_{3}(h) V_{M}^{2}(t)+O_{4}(h),
$$

where all $O_{i}(h) / h$ remain bounded in $M$ and $h$. Now

$$
\begin{aligned}
& E\left\{\left(V_{M}(t)-V_{M}\left(t_{1}\right)\right)^{2}\left(V_{M}(t)-V_{M}\left(t_{2}\right)\right)^{2}\right\} \\
& \quad=E\left\{\left(V_{M}(t)-V_{M}\left(t_{1}\right)\right)^{2} E\left\{\left(V_{M}(t)-V_{M}\left(t_{2}\right)\right)^{2} \| V_{M}(t), V_{M}\left(t_{1}\right)\right\}\right\} \\
& =E\left\{\left(V_{M}(t)-V_{M}\left(t_{1}\right)\right)^{2}\left[V_{M}^{2}(t) O_{5}\left(t_{2}-t\right)+O_{1}\left(t_{2}-t\right)\right]\right\} \\
& =O_{1}\left(t_{2}-t\right) E\left\{\left(V_{M}(t)-V_{M}\left(t_{1}\right)\right)^{2}\right\}+O_{5}\left(t_{2}-t\right) E\left\{\left(V_{M}(t)-V_{M}\left(t_{1}\right)\right)^{2} V_{M}^{2}(t)\right\} .
\end{aligned}
$$

Here $O_{5}(h)=e^{-\beta h G_{1}(M)}-2 e^{-\beta h}+1$, and $O_{5}(h) / h$ remains bounded in $M$ and $h$, provided $M \geqq 10$. Also,

$$
\begin{aligned}
E\left\{\left(V_{M}(t)-V_{M}\left(t_{1}\right)\right)^{2}\right\} & =E\left\{E\left\{\left(V_{M}(t)-V_{M}\left(t_{1}\right)\right)^{2} \| V_{M}\left(t_{1}\right)\right\}\right\} \\
& =E\left\{O_{5}\left(t-t_{1}\right) V_{M}^{2}\left(t_{1}\right)+O_{1}\left(t-t_{1}\right)\right\} \\
& =O_{5}\left(t-t_{1}\right) E\left\{V_{M}^{2}\left(t_{1}\right)\right\}+O_{1}\left(t-t_{1}\right),
\end{aligned}
$$

and

$$
\begin{aligned}
& E\left\{\left(V_{M}(t)-V_{M}\left(t_{1}\right)\right)^{2} V_{M}^{2}(t)\right\} \\
&= E\left\{V_{M}^{4}(t)-2 V_{M}\left(t_{1}\right) V_{M}^{3}(t)+V_{M}^{2}\left(t_{1}\right) V_{M}^{2}(t)\right\} \\
&= E\left\{E\left\{V_{M}^{4}(t) \| V_{M}\left(t_{1}\right)\right\}\right\}-2 E\left\{V_{M}\left(t_{1}\right) E\left\{V_{M}^{3}(t) \| V_{M}\left(t_{1}\right)\right\}\right\} \\
&+E\left\{V_{M}^{2}\left(t_{1}\right) E\left\{V_{M}^{2}(t) \| V_{M}\left(t_{1}\right)\right\}\right\} \\
&= E\left\{e^{-\beta\left(t-t_{1}\right) G_{3}(M)} V_{M}^{4}\left(t_{1}\right)+O_{3}\left(t-t_{1}\right) V_{M}^{2}\left(t_{1}\right)+O_{4}\left(t-t_{1}\right)\right\} \\
&-2 E\left\{e^{-\beta\left(t-t_{1}\right) G_{2}(M)} V_{M}^{4}\left(t_{1}\right)+O_{2}\left(t-t_{1}\right) V_{M}^{2}\left(t_{1}\right)\right\} \\
&+E\left\{e^{-\beta\left(t-t_{1}\right) G_{1}(M)} V_{M}^{4}\left(t_{1}\right)+O_{1}\left(t-t_{1}\right) V_{M}^{2}\left(t_{1}\right)\right\} \\
&= {\left[e^{-\beta\left(t-t_{1}\right) G_{3}(M)}-2 e^{-\beta\left(t-t_{1}\right) G_{2}(M)}+e^{-\beta\left(t-t_{1}\right) G_{1}(M)}\right] E\left\{V_{M}^{4}\left(t_{1}\right)\right\} } \\
&+\left[O_{1}\left(t-t_{1}\right)+O_{2}\left(t-t_{1}\right)+O_{3}\left(t-t_{1}\right)\right] E\left\{V_{M}^{2}\left(t_{1}\right)\right\}+O_{4}\left(t-t_{1}\right)
\end{aligned}
$$

Denote $e^{-\beta h G_{3}(M)}-2 e^{-\beta h G_{2}(M)}+e^{-\beta h G_{1}(M)}$ by $O_{6}(h)$. Again $O_{6}(h) / h$ remains bounded in $M$ and $h$, provided $M \geqq 10$. 
Next we want to show that $E\left\{V_{M}^{2}(t)\right\}$ and $E\left\{V_{M}^{4}(t)\right\}$ are uniformly bounded in $M$ and $t$, for $M \geqq 10$ and $t \geqq 0$. First, we have

$$
E\left\{V_{M}^{2}(t)\right\}=B_{2}(M)(D / \beta)\left[1-e^{-\beta t G_{1}(M)}\right]<2 D / \beta
$$

for all $M \geqq 10$ and all $t \geqq 0$. As to the fourth moment,

$$
\begin{aligned}
E\left\{V_{M}^{4}(t)\right\}= & (D / \beta)^{2}\left[F_{3}(M) B_{2}(M)\left(1-e^{-\beta t G_{3}(M)}\right)\right. \\
& \left.-F_{2}(M) B_{2}(M)\left(e^{-\beta t G_{1}(M)}-e^{-\beta t G_{3}(M)}\right)+F_{4}(M)\left(1-e^{-\beta t G_{3}(M)}\right)\right] \\
\leqq & (D / \beta)^{2}[3+6+1]=(D / \beta)^{2} 10
\end{aligned}
$$

for all $M \geqq 10$ and all $t \geqq 0$.

Using these bounds for $E\left\{V_{M}^{2}\left(t_{1}\right)\right\}$ and $E\left\{V_{M}^{4}\left(t_{1}\right)\right\}$, and substituting (2.5) and (2.6) into (2.4), we get

$$
\begin{aligned}
& E\left\{\left(V_{M}(t)-V_{M}\left(t_{1}\right)\right)^{2}\left(V_{M}(t)-V_{M}\left(t_{2}\right)\right)^{2}\right\} \\
& \leqq O_{1}\left(t_{2}-t\right)\left\{O_{5}\left(t-t_{1}\right) 2 D / \beta+O_{1}\left(t-t_{1}\right)\right\}+O_{5}\left(t_{2}-t\right)\left\{O_{6}\left(t-t_{1}\right) 10(D / \beta)^{2}\right. \\
& \left.+\left[O_{1}\left(t-t_{1}\right)+O_{2}\left(t-t_{1}\right)+O_{3}\left(t-t_{1}\right)\right] 2 D / \beta+O_{4}\left(t-t_{1}\right)\right\} .
\end{aligned}
$$

Take $K_{1}$ so large that $O_{i}(h) \leqq K_{1} h$ for all $i$, all $0 \leqq h \leqq 1$, and all $M \geqq 10$, and let

$$
K=2 K_{1}^{2}+8 K_{1}^{2}(2 D / \beta)+10 K_{1}^{2}(D / \beta)^{2} .
$$

Then we have

$$
E\left\{\left(V_{M}(t)-V_{M}\left(t_{1}\right)\right)^{2}\left(V_{M}(t)-V_{M}\left(t_{2}\right)\right)^{2}\right\} \leqq\left(t_{2}-t\right)\left(t-t_{1}\right) K \leqq K\left(t_{2}-t_{1}\right)^{2}
$$

for all $0 \leqq t_{1} \leqq t \leqq t_{2} \leqq 1$ and all $M \geqq 10$. This concludes the proof of the theorem.

We will next show that removing the atoms after a collision with the molecule does not affect the limit. We are still assuming that the atoms pass through each other and only change velocity as a result of a collision with the molecule.

The first thing to do is to notice that as long as $\left|V_{M}^{\prime}(t)\right|$ stays less than $\frac{1}{4} v(M)$, no atom hits the molecule twice. This is seen from equation (2.1). From that equation we have

$$
\left|v_{2}\right| \geqq \frac{M-1}{M+1}\left|v_{1}\right|-\frac{2 M}{M+1}\left|V_{1}\right|
$$

Thus if $\left|v_{1}\right|=v(M),\left|V_{1}\right|<\frac{1}{4} v(M)$ and $M \geqq 10$, then $\left|v_{2}\right|>\frac{1}{3} v(M)$. That is, as long as $\left|V_{M}^{\prime}(t)\right|$ stays less than $\frac{1}{4} v(M)$, any atom which hits the molecule will bounce away with a speed at least $\frac{1}{3} v(M)$ and hence cannot hit again until $\left|V_{M}^{\prime}(t)\right|$ gets larger than $\frac{1}{3} v(M)$. Therefore $V_{M}(t)$ and $V_{M}^{\prime}(t)$ behave in exactly the same way as long as they both stay less than $\frac{1}{4} v(M)$ in absolute value.

Hence if we define the open set $G_{M} \subset D[0,1]$ by $G_{M}=\left\{f: \sup _{0 \leqq t \leqq 1}|f(t)|<\frac{1}{4} v(M)\right\}$, then for every Borel set $A \subset D[0,1]$ we have

$$
P_{M}\left(G_{M} \cap A\right)=P_{M}^{\prime}\left(G_{M} \cap A\right) .
$$

We are now ready to prove one of our main results. 
2.7 TheOREM. Let $v(M)$ and $\lambda(M)$ be as in Theorem 2.1 and let $P_{M}^{\prime}$ and $P^{\prime}$ be as in the first paragraphs of this section. Then as $M$ goes to infinity, $P_{M}^{\prime}$ converges weakly to $P^{\prime}$.

Proof. It will suffice to show that for every open set $G \subset D[0,1]$

$$
\liminf _{M \rightarrow \infty} P_{M}^{\prime}(G) \geqq P^{\prime}(G) \text {. }
$$

To begin with, $P_{M}^{\prime}(G) \geqq P_{M}^{\prime}\left(G \cap G_{M}\right)=P_{M}\left(G \cap G_{M}\right) \geqq P_{M}\left(G \cap G_{N}\right)$ if $M \geqq N$. We already proved in Theorem 2.1 that $P_{M}$ converges weakly to $P\left(=P^{\prime}\right)$; hence,

$$
\liminf _{M \rightarrow \infty} P_{M}\left(G \cap G_{N}\right) \geqq P^{\prime}\left(G \cap G_{N}\right) .
$$

Therefore,

$$
\liminf _{M \rightarrow \infty} P_{M}^{\prime}(G) \geqq \liminf _{M \rightarrow \infty} P_{M}\left(G \cap G_{N}\right) \geqq P^{\prime}\left(G \cap G_{N}\right),
$$

and the proof is completed by letting $N$ go to infinity.

We conclude by proving Theorem 1.2. For this purpose we define the continuous function

$$
T: D[0,1] \rightarrow C[0,1] \text { by } T f(t)=\int_{0}^{t} f(s) d s .
$$

It is clear that $T V_{M}^{\prime}(t)$ is the position of the molecule at time $t$. For each $M, T$ induces a measure, $\nu_{M}$, on $C[0,1]$ by the formula $\nu_{M}(A)=P_{M}^{\prime}\left(T^{-1}(A)\right)$. Define $v$ to be the measure $\nu(A)=P^{\prime}\left(T^{-1}(A)\right)$. Then $\nu$ is the measure corresponding to the Ornstein-Uhlenbeck position process.

2.8 THEOREM. Under the hypotheses of Theorem $2.7 \nu_{M}$ converges weakly to $\nu$ as $M$ goes to infinity.

Proof. Let $f$ be any bounded continuous real valued function on $C[0,1]$. It will suffice to show that

$$
\int f d \nu_{M} \rightarrow \int f d \nu \text { as } M \rightarrow \infty
$$

Making a change of variable, we see that

$$
\int f d \nu_{M}=\int f \circ T d P_{M}^{\prime} \text { and } \int f d \nu=\int f \circ T d P^{\prime} .
$$

Since $T$ is continuous, $f \circ T$ is a bounded continuous real valued function on $D[0,1]$; and the theorem follows, since $P_{M}^{\prime}$ converges weakly to $P^{\prime}$.

\section{BIBLIOGRAPHY}

1. P. Billingsley, Convergence of probability measures, Wiley, New York, 1968.

2. R. L. Dobrušin, On Poisson's law for distributions of particles in space, Ukrain. Math. Ž. 8 (1956), 127-134. (Russian) 
3. T. E. Harris, Diffusions with "collisions" between particles, J. Appl. Probability 2 (1965), 322-338.

4. R. Holley, The motion of a heavy particle in an infinite one dimensional gas of hard spheres, Thesis, Cornell Univ., Ithaca, N. Y., 1969.

5. D. W. Jepsen, Dynamics of a simple many-body system of hard rods, J. Mathematical Phys. 6 (1965), 405-413.

6. E. Nelson, Dynamical theories of Brownian motion, Princeton Univ. Press, Princeton, N. J., 1967.

7. A. Renyi, On an extremal property of the Poisson process, Ann. Inst. Statist. Math. 16 (1964), 129-133.

8. F. Spitzer, Uniform motion with elastic collision of an infinite particle system, J. Math. Mech. 18 (1969), 973-989.

9. C. Stone, On a theorem of Dobrushin, Ann. Math. Statist. 39 (1968), 1391-1402.

\section{CORNELL UNIVERSITY,}

ITHACA, NeW YoRK 